HAEMOGLOBIN

\section{Monomers Monitored}

from our Molecular Biology Correspondent

THE nature and strength of subunit interactions in proteins has an intimate bearing on the interconversion between states of high and low ligand affinity, which lies at the heart of control of activity. Here, as so often, haemoglobin is the archetype. That the tetramer is in dissociation equilibrium with dimer was established in the very dawn of protein history by the old masters, Svedberg and Pedersen. More recently debate has centred on whether, at sufficiently low concentration, the dimer will in turn give rise to monomer. The general view was that at the lowest observable protein concentrations the monomer remains below the level of detectability. Schachman and Edelstein, on the other hand, reported substantial dissociation into monomers, on the basis of sedimentation equilibrium measurements with scanner optics. This conclusion has now been repudiated by Kellett and Schachman (J. Mol. Biol., 59, 387; 1971).

Scanner optics for this system offer the advantage that by operating at the wavelength of the very intense Soret absorption band of the haemoglobin, measurements can be made at concentrations as low as about $10^{-8} \mathrm{M}$ in subunits. Unfortunately, oxyhaemoglobin in such dilute solution is prone to oxidize to the ferric form, which then denatures, with irreversible formation of monomeric chains. This seems to have been the cause of the previous error. Kellett and Schachman's re-evaluation was made possible by the remarkable observation that methaemoglobin formation is inhibited by the presence of EDTA, which one can only suppose to function by sequestering traces of heavy metals that would otherwise catalyse the oxidation reaction. At all events, under these conditions the equilibrium distribution in the ultracentrifuge reveals that only oxyhaemoglobin dimer is present, and the same is true of other stable derivatives, such as carboxyhaemoglobin and methaemoglobin cyanide and azide. It may be noted that Bucci (ibid., 55, 401; 1971) also recently concluded that at concentrations down to $10^{-8} \mathrm{M}$, oxyhaemoglobin gave rise to no significant amount of monomer, for a complement fixation technique, sensitive at such low levels, revealed the absence of any hybridization between two different molecular species, such as would occur were there a finite degree of dissociation beyond the symmetrical dimer $(\alpha \beta)$ stage.

In an accompanying article, Kellett (ibid., 59, 401; 1971) tackles the much more demanding problem of dissocia- tion of oxy and deoxyhaemoglobin under the influence of high salt concentrations. The difficulties are both experimental, in regard to the anaerobic manipulation of the deoxygenated solutions, and theoretical, in terms of the thermodynamics of the threecomponent system of protein, water and salt. Kellett has devised an analytical procedure which yields the dissociation constant for the dimertetramer equilibrium in terms of a buoyant weight, extracted directly from the sedimentation distribution. The upshot is that whereas the dissociation of oxyhaemoglobin to dimers (though not monomers) is strongly promoted by salt, deoxyhaemoglobin remains tetrameric even at the lowest observable concentrations in $2 \mathrm{M}$ sodium chloride or iodide. Qualitatively the finding of such resistance of the unliganded protein to dissociation is not new, but Kellett's results make it clear that its dissociation constant is not less than three orders of magnitude lower in 2 $M$ sodium chloride than that of oxyhaemoglobin. Evidently then, resistance to dissociation is a feature of the "constrained" (low-affinity) structural state. This has a number of implications in relation to functional schemes that have been propounded, and also explains the apparently anomalous failure of the cooperativity (Hill con- stant) to diminish in high salt, when, it was thought, the protein was dissociated to dimers.

It is interesting to find that some putative evidence for haemoglobin monomers in a quite different context has now been annihilated. The haemoglobin-binding protein of serum, haptoglobin, attaches to haemoglobin at sites on the $\alpha$-chains. Nagel and Gibson found that when haptoglobin was partly saturated with haemoglobin $\alpha$ chains, $\beta$-chains were taken up, and this process occurred at a faster rate than when whole haemoglobin was added in place of $\beta$-chains. This result was put down to a slow dissociation of the tetramer, by way of the known $\alpha \beta$ dimers, to single $\beta$-chains, which then rapidly associated with the haptoglobin-bound $\alpha$-chains. Boyd, Smith and Andrews (Canad. J. Biochem., 49, $891 ; 1971)$ prepared the $\alpha$-chainhaptoglobin complex, and added to it an excess of mouse haemoglobin. By gel filtration they then separated out of the mixture, first, a haemoglobinhaptoglobin stoichiometric complex, second, mouse haemoglobin, and, finally, human $\alpha$-chains. Evidently then, the initially bound $\alpha$-chains are displaced. Now it is known that mouse and human haemoglobins hybridize satisfactorily. Boyd et al. conclude therefore that monomers do

\title{
Supermassive Rotors and Active Galactic Nuclei
}

A LONG standing model for quasars and active galaxies postulates the presence of supermassive differentially rotating stars, supported by radiation pressure, at the centres of these objects. In spite of other suggestions, including the possibility of pulsar-like emission from a supermassive rotating object, this relatively simple model remains a likely explanation for the energy source in quasars and galaxies. But the model is only superficially simple-although it is clear from the most naive calculations that a supermassive rotating disk satisfies the broad requirements of the primary energy supply in highly energetic sources, quantitative calculations concerning the evolution of such disks remain inadequate. It is only within the past two years that it has been shown that disks of this kind are unstable against fragmentation, and although this complicates further calculations the prospect raises some interesting conjectures regarding the ultimate fate of the disks. E. E. Salpeter, of Cornell University, has now provided an outline of the successive fragmentations which should accompany the slow evolution of a supermassive star. This approach, presented in next Monday's Nature Physical Science, raises several questions which point the way for further development of the model.

It seems that, so long as the disk is radiation dominated, fragmentation must occur. This process will be repeated in the subdisks formed, until small objects supported by hydrodynamic forces are formed. Because the descendants of the original disk will form a hierarchical grouping after several fragmentations, radiation escaping from the system will suffer successive redshifts as it leaves first the subgroup from which it originates, then the group of subgroups in which that subgroup lies, and so on.

One source of electromagnetic radiation could be pulsar-like activity, which would occur just before the fragmentation of any disk which carries a mag netic field.

The major difficulties in extrapolating this simple picture to more realistic models concern differential rotation and the detailed nature of the gravitational and pulsar radiation emitted. Gravitational radiation, for example, might result in selective changes in the orbits of the fragments until a stable, symmetric pattern with no gravitational radiation is formed. 